\title{
POLAR: Gamma-Ray Burst Polarimetry onboard the Chinese Spacelab
}

\author{
Merlin Kole on behalf of the POLAR collaboration*t \\ DPNC, University of Geneva \\ E-mail: merlin.kole@unige.ch
}

\begin{abstract}
Polarimetry is a powerful tool to study the emission processes involved in high energy astrophysical events such as Gamma-Ray Bursts (GRBs). Despite the wealth of information which can be extracted from such measurements few have been performed successfully thus far. POLAR is a novel space-borne Compton polarimeter foreseen to be launched in 2016 on the Chinese spacelab Tian-Gong 2. The instrument is designed for dedicated measurements of the hard X-ray polarisation of the prompt emission of GRBs in the energy range $50-500 \mathrm{keV}$. The polarisation degree and angle of a photon flux can be extracted by measuring the Compton scattering angles when the photons interact in a detector. The Compton scattering angles of the incoming photons are measured in POLAR using a finely segmented plastic scintillator array consisting of 1600 bars. The bars have a surface area of 6 by $6 \mathrm{~mm}$ and a length of $176 \mathrm{~mm}$ and are read out in groups of 64 by 25 flat-panel multi-anode photomultipliers. Due to its large granularity POLAR can measure the photon interaction locations, and therefore the scattering angles, with a high precision resulting in a relatively high modulation factor. The instrument furthermore has a relatively large effective area and a field of view of $1 / 3$ of the sky thereby optimising it for studying GRBs. The instrument was shown through Geant 4 simulations to be capable of performing measurements with a minimum detectable polarisation below $10 \%$ for several GRBs per year. The flight model has recently been constructed and was tested extensively in recent months. The results from the instrument calibration measurements, performed using a polarised beam at a synchrotron facility, and the results from the flight acceptance tests will be presented along with the future prospects.
\end{abstract}

The 34th International Cosmic Ray Conference,

30 July- 6 August, 2015

The Hague, The Netherlands

\footnotetext{
${ }^{*}$ Speaker.

${ }^{\dagger}$ As presented: on isdc.unige.ch/polar/
} 


\section{Introduction}

To date polarimetry remains a relatively unexplored field of high energy astrophysics. Whereas the flux, spectral and timing measurements in the $\mathrm{X} / \gamma$-ray energy range have been performed for a large variety of astrophysical sources, the two remaining 2 parameters of the emission, the polarisation degree and angle, remain largely unprobed to date. This while these two variables hold valuable information on the mechanisms responsible for the emission of the photons and the environments in which they were emitted. Polarimetry is therefore a powerful tool to study high energy astrophysical environments such as neutron starts, X-ray binary systems, active galactic nuclei, solar flares and Gamma Ray Bursts (GRBs).

In recent years a large number of new missions has been proposed dedicated to measure the polarimetric parameters and thereby opening up a new field in high energy astrophysics. Currently several new dedicated polarimeters are under construction, some examples are GRAPE [1], XCalibur [2], XIPE [3] and Tsubame [4] while several smaller scale missions, such as GAP [5][6] and PoGOLite [7] have performed measurements in recent years. Furthermore a number of instruments without a dedicated polarimeter have managed to perform polarimetric measurements of astrophysical sources in the $\mathrm{X} / \gamma$ ray energy range. Examples of this are the IBIS and SPI instruments on the Integral mission which for example both published polarimetric measurements of the Crab pulsar [8] [9]. These measurements have however in generally been non-constraining.

The POLAR instrument [10] is a new dedicated polarimeter foreseen to be placed on the Chinese Tian-Gong Space Lab during 2016. The instrument uses a highly segmented scintillator array optimized for performing polarimetry measurements in the 50-500 keV energy range, while its wide field of view allows it to study GRBs. In this paper the scientific goal of the instrument will be discussed first, followed by a detailed description of the polarimeter. This is followed by the first results from calibration measurements and environmental tests of the POLAR flight model. Finally the future prospects of the instrument, which is foreseen to be launched during the summer of 2016 will be presented.

\section{GRBs}

From an observers point of view GRBs are strong short flashes of $\mathrm{X} / \gamma$-rays, referred to as the prompt-phase, followed by a longer lasting afterglow which can be measured at lower energies. The length of the prompt-phase emission varies from seconds to minutes and two distinct populations can be seen in the length distribution. The short burst are theorised to originate from the merger of two compact objects such as neutron starts, whereas the longer bursts are thought to originate from the collapse of massive stars. During both kinds of GRBs the emission in the prompt phase is thought to originate from two jets, as a result bursts can only be measured when the jet is within the line of sight of the observer.

Astrophysical jets, especially those from GRBs are poorly understood. Polarimetric measurements are however capable of probing several aspects of the jets and can therefore be used to answer some of the many questions concerning the gamma-ray emission mechanisms and environments of GRBs. Questions which spectral analysis performed, for example by the two experiments on the Fermi satellite [11], have not been able to answer. 
An example of a property of the emission region which can be understood using polarimetric measurements is the order of the magnetic field within the emission jet. A jet with a strong magnetic field will result in the emission of high energy photons through synchrotron emission resulting in high polarisation degrees. The observer will only observe a part of the jet. In the case of a highly ordered magnetic field the field in the observed region will be relatively constant, resulting in a constant polarisation angle emitted within this region. The observer will therefore measure a high polarisation degree, which can be as high as $50 \%$ [12]. The exact polarisation degree will depend on the viewing angle with respect to the jet, which in the case of a highly ordered magnetic field results in an average polarisation degree of $40 \%$ within a sample of GRBs. In case of a non ordered magnetic field the polarisation angle will vary within the observed region of the jet resulting in a low observed polarisation degree. Models with a non-ordered magnetic field within the jet therefore predict a distribution of the polarisation degree of a GRB sample to peak close to $0 \%$.

A second example of a property of GRBs which is not yet understood that can be resolved using polarimetric measurements is the internal structure of the emission jet. Different models exist regarding the structure some of which predict an axisymmetric structure, whereas others predict a jet consisting of several mini-jets. An axisymmetric model makes the strong prediction that the polarisation degree of the emission can only change during the observation by $90^{\circ}$, or it can not change at all [13]. For models with mini-jets the observer will at any time during the burst observe the strongest mini-jet in the line of sight, as the different jets will likely emit gamma rays with different polarisation angles the observed polarisation angle can vary randomly during such a burst. Polarisation measurements of a sample of GRBs can therefore be used to distinguish between these two model groups. Thus far the GAP instrument has measured one strong burst during which there is strong evidence that the angle varies during the burst by $90^{\circ}$ [5], however, the error on the angle and its change during the burst are rather large and more measurements are required to distinguish between the different models.

These different examples show that measurements of the polarisation angle and degree of a sample of GRBs will result in a deeper knowledge about GRBs. For this purpose the POLAR detector was developed. This polarimeter has a wide field of view, a large sensitivity to polarisation and a relatively large effective area, allowing it to accurately measure the polarisation of several GRBs per year.

\section{POLAR}

POLAR is a novel compact space-borne Compton polarimeter which uses 25 flat-panel multianode photomultipliers (Hamamatsu H8500) modules. Each module has 64 channels each of which reads out an individual plastic scintillator bar. The PMTs are read out using individual Front-End Electronics (FEE). The 25 FEEs are in turn controlled using 3 FPGAs (and 3 additional FPGAs serving as a back-up). The scintillator bars are wrapped in Vikuiti to improve the light yield and to reduce the optical cross-talk between channels. A single module is shown in figure 1, while the full flight model is shown in figure 2. Photons entering the scintillator array can undergo Compton scattering in one of the scintillator bars, followed by a second Compton scattering interaction or photoelectric absorption in a secondary bar. This is schematically shown in figure 3. Using the position of the two scintillator bars the azimuthal Compton scattering angle of the incoming photon 
can be determined. This angle holds information on the initial polarisation angle of the photon. This is described in the Klein-Nishina equation [14]:

$$
\frac{\mathrm{d} \sigma}{\mathrm{d} \Omega}=\frac{\mathrm{r}_{0}^{2}}{2} \frac{\mathrm{E}^{\prime 2}}{\mathrm{E}^{2}}\left(\frac{\mathrm{E}^{\prime}}{\mathrm{E}}+\frac{\mathrm{E}}{\mathrm{E}^{\prime}}-2 \sin ^{2} \theta \cos ^{2} \phi\right) .
$$

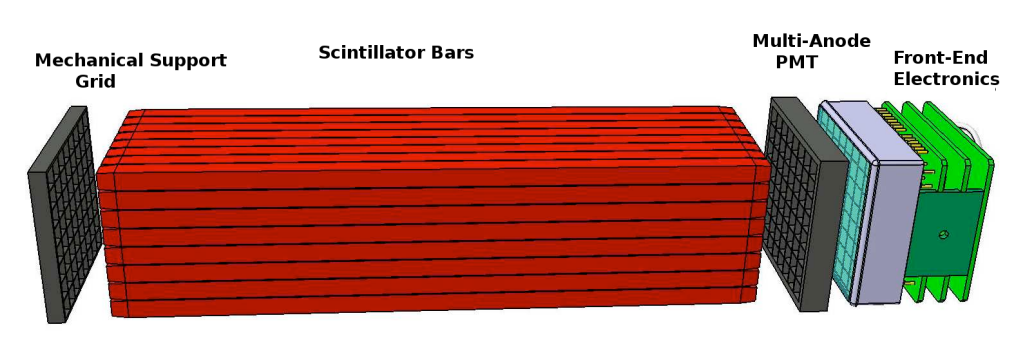

Figure 1: Schematic representation of a single module of POLAR. The scintillator bars are shown in red, together with the MAPMT, the front-end electronics and the mechanical support structure.

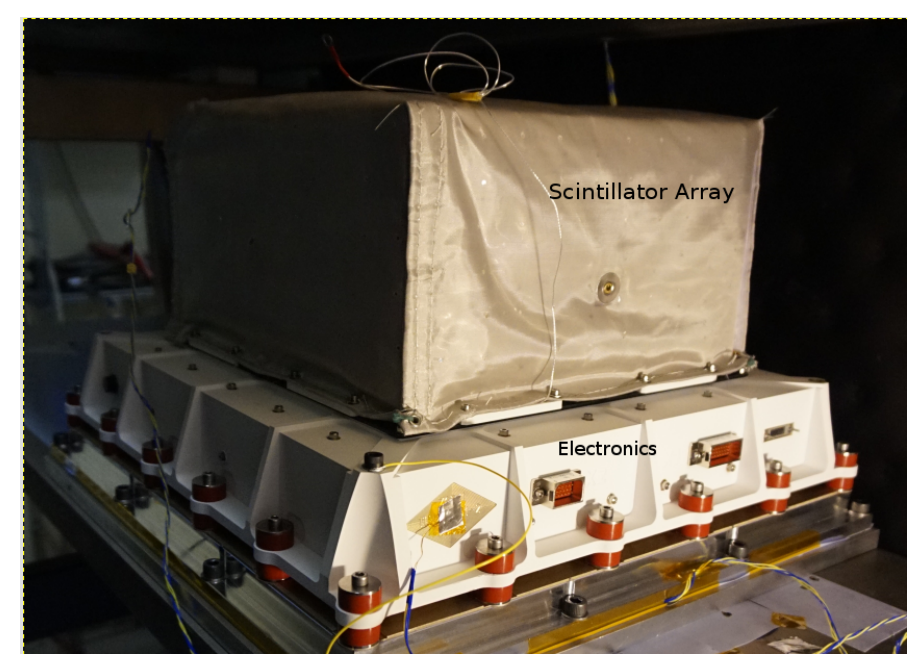

Figure 2: The POLAR flight model during thermal vacuum tests. The electroncs is contained in the white painted alumunium structure while the scintillator array is placed in a carbon structure covered with a multilayer insulator.

Here $r_{0}^{2}$ is the classical electron radius, $E$ is the initial photon energy, $E^{\prime}$ the final photon energy, $\theta$ the polar scattering angle and $\phi$ the azimuthal Compton scattering angle with respect to the polarisation vector. This is visualised in figure 3. The $\cos ^{2} \phi$ term in this equation results in a polarisation dependent cross section and a higher probability for scattering perpendicular to the polarisation vector. For a polarised photon flux Compton scattering on a target this results in a nonuniform distribution of the azimuthal scattering angles. The distribution of azimuthal scattering angles can be described by a function of the form

$$
f(\eta)=A\left(1+\mu \cos \left(2\left(\eta-\eta_{0}\right)+\pi\right)\right.
$$

In which $\eta_{0}$ represents the polarisation angle and $\mu$ is referred to as the modulation factor. The polarisation degree can be retrieved from $\mu$ by dividing it by $\mu_{100}$ which is the instrument's 
response to a $100 \%$ polarised beam. As the value of $\mu_{100}$ is instrument and energy dependent this value has to be acquired using both calibration measurements and simulations. The accuracy with which a polarisation measurement can be performed can be expressed using Minimal Detectable Polarisation (MDP). This parameter represents the minimum degree of polarisation required in order to be able to confirm through measurement that the observed signal is polarised within a set confidence level.

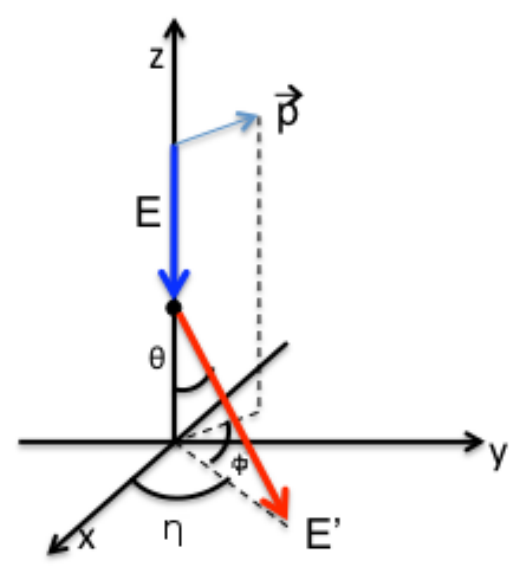

Figure 3: Schematic representation of the Compton scattering process. The incoming photon (blue; energy E, polarisation $\vec{p}$ ) scatters off an electron with a polar angle $\theta$ from its original trajectory. $\phi$ is the angle between the polarisation vector of the incoming photon and the azimuthal scattering angle $\eta$.

The interaction location within the scintillator bars cannot be determined. Therefore the precision with which the polarisation angle can be measured, which is reflected in the value of $\mu_{100}$, is highly dependent on the size of the scintillator bars. For this purpose POLAR makes use of a finely segmented scintillator array in which each scintillator has a size of $6 \times 6 \times 176 \mathrm{~mm}$ resulting in $\mu_{100}$ values exceeding $40 \%$ [15]. The choice for light weight, low-Z plastic scintillator results in a high cross section for Compton scattering in the POLAR energy range which is $50-500 \mathrm{keV}$ while keeping the mass of the full instrument around $30 \mathrm{~kg}$. The large uniform detector area furthermore results in both a large effective area and a relatively small dependence of $\mu_{100}$ on the incoming photon angle with respect to zenith. As a result the instrument can observe a large portion of the sky and is therefore optimised for performing polarisation measurements of GRBs.

\section{Calibration Tests}

During the spring 2015 the flight model of POLAR was constructed and in May 2015 the full instrument was calibrated at the European Synchrotron Facility (ESRF) in Grenoble in France using a $100 \%$ polarised photon beam with a maximum energy of $140 \mathrm{keV}$. The setup at this test can be seen in figure 4. In order to simulate a GRB observation an equal irradiation of the full surface is required, for this purpose the full surface of POLAR was scanned using the polarised photon 
beam, which has a size of $0.6 \times 0.6 \mathrm{~mm}$, using two motors. Additionally similar measurements were performed with different beam energies of 60,80 and $110 \mathrm{keV}$ and scans were performed where the surface of POLAR was placed at angles of $30^{\circ}$ and $60^{\circ}$ in order to study the instruments sensitivity to off-axis GRBs. Lastly each scan was performed twice, where POLAR was rotated by $90^{\circ}$ with respect to the polarisation vector in between measurements. By combining the data from the two measurements the response to an unpolarised beam can be studied.

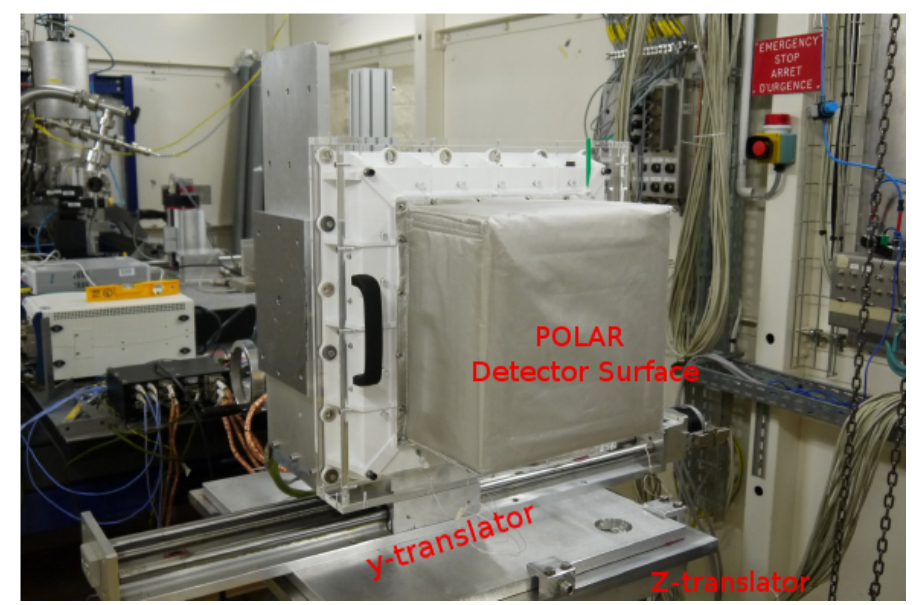

Figure 4: The POLAR flight model during the ESRF beam tests. In this setup the detector surface is perpendicular to the beam. The polarimeter is placed 2 translators which move it with respect to the beam.

The first results of the analysis of the $140 \mathrm{keV}$, on-axis illumination can be seen in figure 5. This modulation curve shows a $\mu_{100}$ value for $140 \mathrm{keV}$ photons of $\approx 40 \%$ which is compatible with the values previously acquired from simulation [15], while for an unpolarised beam no obvious modulation is observed. Further optimisation of the analysis is however required. Several aspects of the analysis will result in the removal of systematic effects induced in the modulation, an example of this is the variations of the intensity with time of the beam. The final results of the ESRF data will be the subject of a future paper.

\section{Future Prospects}

During late June and early July of 2015 the POLAR flight model underwent the flight acceptance tests both at the University of Geneva and at the SERMS facility in Terni, Italy. The flight model underwent thermal cycling tests, sinusoidal vibration tests, random vibration tests, pyroshock tests and finally a thermal vacuum test. As the full flight model did not sustain any damage during these tests it passed all these tests. The flight model will be shipped to IHEP in Beijing, China in July 2015, where it will undergo the final burn-in test in August of 2015. Subsequent to the completion of the burn-in test final compatibility tests with the Tian-Gong 2, will take place at GESSA in China during the end of 2015. The launch of POLAR, which will be together with that of Tian-Gong 2 is currently foreseen to take place during the summer of 2016. 


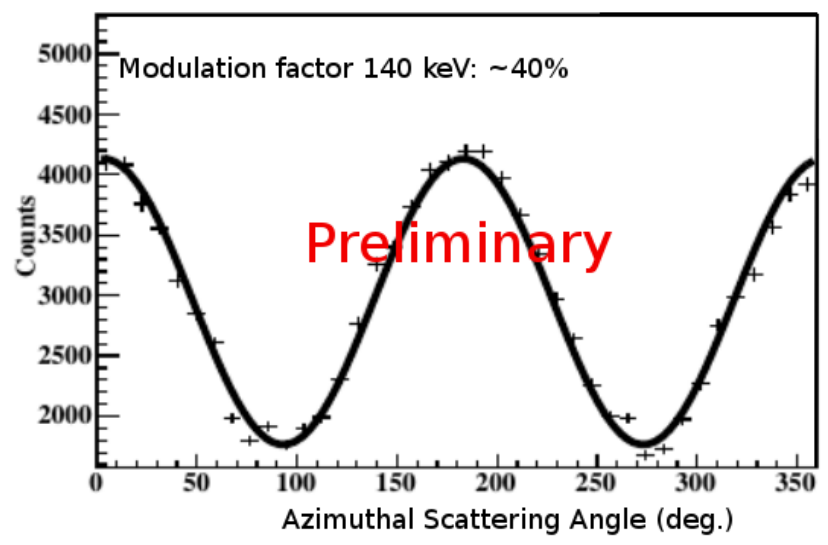

Figure 5: The preliminary results of the modulation factor measured for a $100 \%$ polarised beam of $140 \mathrm{keV}$ at ESRF.

\section{Conclusions}

POLAR is a novel polarimeter designed to measure the polarisation of the prompt emission of GRBs in the $50-500 \mathrm{keV}$ energy range. The detector will be mounted on the Chinese Space Lab, the Tian-Gong 2, with a lifetime of 2 years. The full flight model has been tested at ESRF using a $100 \%$ polarised beam at energies of $140,110,80$ and $60 \mathrm{keV}$ at 3 different incoming angles. The preliminary results of this tests are compatible with previously published simulation results and therefore confirm the relatively high sensitivity of POLAR to a polarised beam, making it capable of measuring the polarisation of several GRBs during its lifetime with an MDP of 10\%. The flight model has furthermore undergone most of the flight acceptance tests successfully and is currently in the final stages of preparation for launch foreseen to be the summer of 2016.

\section{Acknowledgements}

The authors acknowledge ESRF and specifically the personnel of ID11 for their support during the calibration tests of POLAR. Furthermore the authors acknowledge the personnel of of SERMS s.r.l. in Terni for their valuable assistance during the acceptance tests of the POLAR flight model.

\section{References}

[1] P.F. Bloser et al. 2008, arXiv:0812.0782v1

[2] Q. Guo et al. ApJ. 41 (2013) 63

[3] P. Soffitta et al. 2013, arXiv:1309.6995

[4] S. Kurita et al. 2015, arXiv:1503.01975

[5] D. Yonetoku et al. ApJ. 743 (2011) 30

[6] D. Yonetoku et al. ApJ. 758 (2012) 1 
[7] M. Kole, Doctoral Thesis, KTH - Royal Institute of Technology, Stockholm (2014). http://kth.diva-portal.org

[8] M. Chauvin et al. ApJ. 769 (2013) 137

[9] M. Forot et al. ApJ. 688 (2008) 29

[10] N. Produit et al., Nucl. Instr. and Meth. A 550 (2005) 616.

[11] A. A. Abdo et al. ApJ. Sup. Ser. 187 (2010) 460

[12] M. Luytikov et al., ApJ. 597 (2003) 998

[13] C. Lundman et al. MNRAS 428 (2013) 2430

[14] O. Klein, Y. Nishina, Z. Phys. 52 (1929) 853

[15] E. Suarez, Doctoral Thesis, Univ. of Geneva 2010, at http://archive-ouverte.unige.ch/unige:6889 